\title{
The "Climb" Towards Minimal Disease Activity in Psoriatic Arthritis
}

\author{
Ennio Lubrano (D) - Silvia Scriffignano (D) - Fabio Massimo Perrotta
}

Received: April 22, 2021 / Accepted: June 23, 2021 / Published online: July 24, 2021

(C) The Author(s) 2021

\begin{abstract}
Introduction: Minimal disease activity (MDA) is a validated outcome measure in psoriatic arthritis (PsA) defining a low disease activity state with a cutoff of $5 / 7$. The main aim of the study was to look at the MDA divided into in the seven cutoffs, analyzing the more frequently achieved domains. The relationship between MDA, PASS, PsAID, DAPSA, and the PhGA in all cutoffs was also evaluated.

Methods: Cross-sectional analysis on PsA patients satisfying CASPAR criteria. An assessment of disease activity, treatment target, function, and impact of disease was performed. Patients achieving MDA were compared to patients not achieving MDA in order to evaluate the most frequent domain found.
\end{abstract}

Ennio Lubrano and Silvia Scriffignano contributed equally to this work.

E. Lubrano $(\varangle) \cdot S$. Scriffignano - F. M. Perrotta Dipartimento di Medicina e Scienze, Della Salute "Vincenzo Tiberio", Università degli Studi del Molise, Via Giovanni Paolo II, C/da Tappino, 86100 Campobasso, Italy

e-mail: enniolubrano@hotmail.com
Results: Ninety-three PsA patients were enrolled. MDA was satisfied in $44 / 93$, while in 47 MDA ranged from $1 / 7$ to $4 / 7$. Among the seven domains, Leeds Enthesitis Index (LEI) was the most frequent domain found in all patients. In those not in MDA, BSA $\leq 3(70 \%)$ and swollen joints count $\leq 1(68 \%)$ were also well represented. The domains with a lower percentage of patients not in MDA were $\mathrm{HAQ}-\mathrm{DI} \leq 0.5$ $(38.8 \%)$, tender joint count $\leq 1 \quad(23 \%)$, PtGA $\leq 20(4.2 \%)$ and VAS pain $\leq 15 \mathrm{~mm}(2 \%)$. There was a growing trend, from MDA $1 / 7$ to MDA 7/7 in the percentage of patients in PASS yes, in PsAID $\leq 4$, and in DAPSA $\leq 14$.

Conclusions: The present study detailed the domains more achieved also in those patients not in MDA showing that "physician-driven" domains are more frequently achieved in our patients.

Keywords: Psoriatic arthritis; Minimal disease activity; Assessment; Outcome measures 


\section{Key Summary Points}

PsA is an inflammatory chronic disease with different clinical manifestations.

Minimal disease activity (MDA) is an achievable treatment target for PsA patients. However, even when MDA is not achieved, the disease state could be very different when the cut-off of 4 out of 7 criteria is reached, compared to a cut-off of 1 out of 7 .

Our study demonstrated that some domains are frequently achieved even in patients not in MDA. In particular, swollen joints, enthesitis (by LEI) and $\mathrm{BSA} \leq 3$ are frequently achieved even in those not in MDA.

The study showed that "physician-driven" domains are more frequently achieved in all enrolled patients. Moreover, a strong correlation was found with other outcome measures throughout the seven domains.

\section{INTRODUCTION}

Psoriatic arthritis (PsA) is a chronic inflammatory disease characterized by a variable clinical course $[1,2]$. In the last 10 years, the achievement of clinical remission or low disease activity have been proposed as treatment targets for PsA patients [3, 4]. In particular, minimal disease activity (MDA) is a categorical and validated measure in PsA as treatment response criteria to capture a disease state: patients are in MDA when they satisfy 5/7 of previously published criteria [4]. More recently, a stringent definition of remission (very low disease activity, VLDA), in which all $7 / 7$ criteria had to be satisfied, has been proposed [5].

MDA has been useful to define the disease state in randomized controlled trials [6] and in real-world evidence studies [7, 8]. However, even when MDA is not achieved, the disease state could be very different when the cutoff of 4 out of 7 criteria is reached, comparing to a cutoff of 1 out of 7 . This could be interesting to be assessed in real life because the achievement of a cutoff of $4 / 7$ criteria has, probably, a different clinical meaning compared to the achievement of only $1 / 7$ criteria.

As potential lack in this field is the assessment of which domains are more frequently achieved in those patients non reaching the MDA 5/7 onwards 7/7. In other words, what are the more frequently missed/achieved domains in patients that do not achieve MDA?

Therefore, the aim of this clinical study was to look at the MDA divided into the seven cutoffs (from $1 / 7$ to $7 / 7$ ) and analyzing which domains were more frequently achieved, in particular in those not satisfying the MDA, namely from $1 / 7$ to $4 / 7$, in a group of PsA patients, as a "climb" towards MDA. A secondary aim was to assess the relationship between MDA, categorized into seven cutoffs, with the Patient Acceptable Symptoms State (PASS) [9], the Psoriatic Arthritis Impact of the Disease (PsAID) [10], the Disease Activity for Psoriatic Arthritis (DAPSA) [11], and the Physician Global Assessment (PhGA).

\section{METHODS}

\section{Patient Selection}

In this cross-sectional analysis of a longitudinal cohort, patients were enrolled at the Rheumatology Unit, Department of Medicine and Health Science-University of Molise. From the June 30, 2019 until December 31, 2019, all PsA patients who were on at least 6-month followup treatment with conventional synthetics (cs) and biologic (b) disease-modifying anti-rheumatic drugs (DMARDs) were considered potentially eligible for the study.

Inclusion criteria were:

(1) PsA classified with the ClASsification criteria for Psoriatic ARthritis (CASPAR) criteria [12],

(2) Age $\geq 18$ years, 
(3) AT least 6 months of follow-up at the study visit,

(4) Stable treatment with a csDMARDs or bDMARDs for at least 6 months.

\section{Data Collection}

Patients' data collection included a medical history, physical examination, current use of medications, and laboratory assessment. Demographics and disease characteristics including age, sex, body mass index, and disease duration were taken into account. The clinical assessment encompassed the number of tender and swollen joints $(68 / 66)$, enthesitis by the Leeds Enthesitis Index (LEI) [13], and dactylitis. Psoriasis was quantified by the body surface area (BSA) [14]. The Health Assessment Questionnaire-Disability Index (HAQ-DI) [15] was used to assess function. Patient Global Assessment (PtGA) and pain on Visual Analogic Scale (VAS) were performed by all patients. PhGA of disease activity on a VAS [16] and C-reactive protein (CRP) were also collected.

\section{MDA, VLDA, DAPSA, PASS, and PsAID}

MDA was defined according to Coates et al. [4]. Patients were considered in MDA when they satisfied $5 / 7$ of the following criteria: tender joint count $\leq 1$; swollen joint count $\leq 1$; BSA $\leq 3$; pain on $\mathrm{VAS} \leq 15 \mathrm{~mm}$; $\mathrm{PtGA} \leq 20 \mathrm{~mm}$; HAQ-DI $\leq 0.5 ; \quad$ LEI $\leq 1$. VLDA was satisfied when all seven criteria were met [5].

DAPSA was calculated by adding the number of tender and swollen joints, pain on VAS, PtGA, and CRP (mg/dl) [17]. The PASS is a single question tool to evaluate the level of symptoms at which patients consider themselves well [9]. The PsAID was also assessed [10].

The study protocol was in compliance with the Declaration of Helsinki; written consent was obtained from each participant. The study was approved by the Institutional Review Board of the University of Molise (protocol no. 0001-092017).

\section{Statistical Analysis}

Statistical analysis was performed using SPSS (version 27). All demographical and clinical characteristics were summarized by using descriptive statistics. Parametric variables were reported by mean \pm standard deviation (SD), and non-parametric ones by median and interquartile range (IQR). Categorical data are shown as number and percentage. Spearman's correlation was used to assess the relationship between MDA divided in the seven cutoffs and DAPSA, PhGA, PsAID. A Spearman's coefficient rho $\leq 0.2=$ very weak, $<0.4=$ weak,$<0.6=$ moderate, $<0.8$ strong and $\leq 1$ very strong correlation, respectively. A significance level was accepted at $p \leq 0.05$.

\section{RESULTS}

\section{Patient characteristics and overall disease activity}

In the study period, 93 PsA satisfied the inclusion criteria and were enrolled. Table 1 shows the main clinical characteristics of the enrolled patients. In particular, in Table 1 are reported the different proportions of patients achieving the different MDA cutoffs; 44/93 (47.3\%) patients were in MDA.

\section{Analysis of Different Domains}

LEI $\leq 1$ was the most frequently achieved domain either in the 47 patients not achieving MDA, or in those 44 patients in MDA. On the other hand, pain on VAS $\leq 15 \mathrm{~mm}$ was the most frequently missed domain in patients achieving or not MDA. Beyond LEI, the other domains frequently achieved in patients not in MDA were $\mathrm{BSA} \leq 3(70 \%)$ and swollen joints count $\leq 1(68 \%)$. On the contrary, the domains with a lower percentage of achievement in patients not in MDA were: HAQ-DI $\leq 0.5$ (38.8\%), tender joint count $\leq 1 \quad(23 \%)$, PtGA $\leq 20(4.2 \%)$, and pain on $\mathrm{VAS} \leq 15 \mathrm{~mm}(2 \%)$. All data are shown in Fig. 1. It also shows the "climb" of MDA, divided into the seven cutoffs. 
Table 1 Demographic and clinical disease characteristics of the 93 PsA patients

\begin{tabular}{|c|c|}
\hline & 93 PsA patients \\
\hline Sex $(\mathrm{M}), n(\%)$ & $61(65.6)$ \\
\hline Age, mean (SD) & $55.3(13.1)$ \\
\hline $\begin{array}{l}\text { Disease duration (months), median } \\
\text { (IQR) }\end{array}$ & $72(24-156)$ \\
\hline Weight (kg), median (IQR) & $75(66-85)$ \\
\hline Height (m), median (IQR) & $1.70(1.64-1.75)$ \\
\hline BMI $\left(\mathrm{kg} / \mathrm{h}^{2}\right)$, median (IQR) & $\begin{array}{l}26.12 \\
\quad(22.85-29.72)\end{array}$ \\
\hline BSA, median (IQR) & $1(0-3)$ \\
\hline \multicolumn{2}{|l|}{ Dactylitis, $n(\%)$} \\
\hline Never & 61 \\
\hline Present & 11 \\
\hline Past & 19 \\
\hline PtGA, median (IQR) & $4(2-6)$ \\
\hline VAS pain, median (IQR) & $4(1-7)$ \\
\hline VAS physician, median (IQR) & $3(1-5)$ \\
\hline $\mathrm{TJ} / 68$, median (IQR) & $1(0-3)$ \\
\hline SwJ/66, median (IQR) & $0(0-1)$ \\
\hline LEI, median (IQR) & $0(0-1)$ \\
\hline CRP (mg/dl), median (IQR) & $0.2(0.18-0.50)$ \\
\hline HAQ-DI, median (IQR) & $\begin{array}{l}0.500 \\
\quad(0.125-0.750)\end{array}$ \\
\hline DAPSA, median (IQR) & $\begin{array}{l}10.40 \\
\quad(4.40-16.83)\end{array}$ \\
\hline PsAID, median (IQR) & $2.60(1.00-4.75)$ \\
\hline PASS yes, $n(\%)$ & $59 / 87(67.8)$ \\
\hline MDA 5/7 n (\%) & $44(47.3)$ \\
\hline \multicolumn{2}{|l|}{$\operatorname{MDA} n(\%)$} \\
\hline $7 / 7$ & $14(15.05)$ \\
\hline $0 / 7$ & $2(2.15)$ \\
\hline $1 / 7$ & $5(5.37)$ \\
\hline $2 / 7$ & $13(14.0)$ \\
\hline $3 / 7$ & $16(17.2)$ \\
\hline
\end{tabular}

Table 1 continued

\begin{tabular}{ll}
\hline & 93 PsA patients \\
\hline $4 / 7$ & $13(14.0)$ \\
$5 / 7$ & $12(12.9)$ \\
$6 / 7$ & $18(19.35)$ \\
Therapy, $n(\%)$ & \\
NSAIDs & $10 / 91(11)$ \\
Oral steroids & $5 / 91(5.5)$ \\
Methotrexate & $16 / 91(17.6)$ \\
Anti-TNF- $\alpha$ & $38 / 91(41.7)$ \\
Anti-IL 17 & $17 / 90(18.9)$ \\
Anti-IL-12/23 & $9 / 90(10)$ \\
Apremilast & $5 / 90(5.5)$ \\
\hline
\end{tabular}

CRP C-reactive protein, $L E I$ Leeds Enthesitis Index, $B S A$ body surface area, $P t G A$ patient global assessment, VAS Visual Analogue Scale, DAPSA disease activity for psoriatic arthritis, PsAID psoriatic arthritis impact of the disease, $P A S S$ patient acceptable symptoms state, $M D A$ minimal disease activity, HAQ-DI Health Assessment Questionnaire-Disability Index, NSAIDs non-steroid anti-inflammatory drugs, anti-TNF- $\alpha$ anti-tumor necrosis factor alfa, $I L$ interleukin, $S D$ standard deviation, $I Q R$ interquartile range

There was a growing trend, from MDA 1/7 to MDA 7/7, in the percentage of patients in PASS yes, in PsAID $\leq 4$ and in DAPSA $\leq 4$ and $\leq 14$. In particular, in those MDA 4/7, more than $80 \%$ were in PASS yes, $75 \%$ in PsAID $\leq 4$ and more than $90 \%$ in DAPSA $\leq 14$.

An inverse correlation (Fig. 2) was found between MDA categories with DAPSA, (rho $=-$ 0.91, $p<0.001)$, PhGA (rho $=-0.76, p<0.001$ ) and PsAID (rho $=-0.78, p<0.001$ ).

\section{DISCUSSION}

To our knowledge, this was the first study to detail the achievement of each single domain included in the MDA and divided by the seven categories. In particular, it seems that some domains are frequently achieved in patients not 


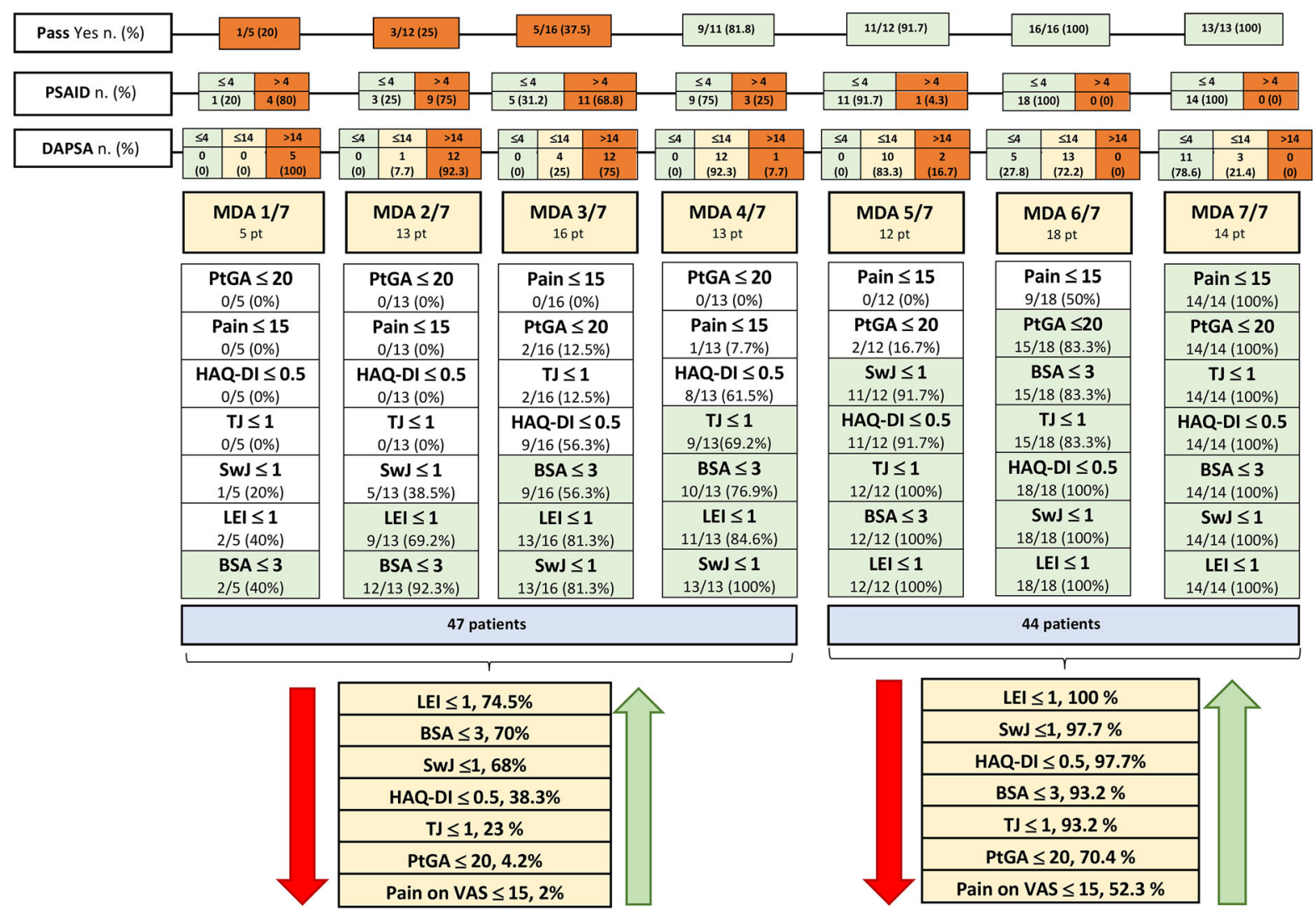

Fig. 1 Numbers and percentage of patients that achieved each MDA domain, divided into the seven cutoffs. For both the 47 patients that did not meet the MDA status (MDA 1/7, 2/7, 3/7,4/7) and the 44 patients in MDA status (MDA5/7, 6/7, 7/7) the cumulative percentage of each MDA domain achieved is shown. PASS patient acceptable symptoms state, PsAID psoriatic arthritis

in MDA, as well as in those in MDA. In fact, in those 47 patients not achieving MDA 5/7, LEI $\leq 1, \mathrm{BSA} \leq 3$ and swollen joints count $\leq 1$ were satisfied in more than $60 \%$. On the other hand, in the same group of 47 patients, there was a drop of the other domains which were ranging from $\mathrm{HAQ}-\mathrm{DI} \leq 0.5$ in $38 \%$ until pain on VAS $\leq 15 \mathrm{~mm}$ in $2 \%$. Overall, this study showed that the "objective" or "physician-driven" domains were more frequently reached in all PsA patients enrolled, MDA or not; on the other hand, the most frequently missed domains are those "patient-driven". Moreover, if we look at those 13 patients in MDA 4/7, 81.8\% were in PASS yes, $75 \%$ in PsAID $\leq 4$ and $92.3 \%$ in DAPSA $\leq 14$. As a further element to consider, impact of the disease, DAPSA disease activity for psoriatic arthritis, $M D A$ minimal disease activity, $L E I$ Leeds Enthesitis Index, BSA body surface area, PtGA Patient Global Assessment, Pain Pain on Visual Analogue Scale, HAQ-DI Health Assessment Questionnaire-Disability Index, $S w J$ swollen joints count, $T J$ tender joints count

the correlation found between MDA with DAPSA, PhGA, and PsAID might support the view of MDA as an instrument capable of complying with disease activity, physician, and patient's perspective throughout the seven cutoffs.

However, the achievement of some treatment targets is not always agreed with the patient's perception of the disease [18] and this is still an unmet need. In fact, it is possible to observe some residual disease activity in PsA patients and this, possibly, might imply some therapeutic decisions [19]. Furthermore, our study showed that residual activity is detectable in PsA patients even in a condition of 


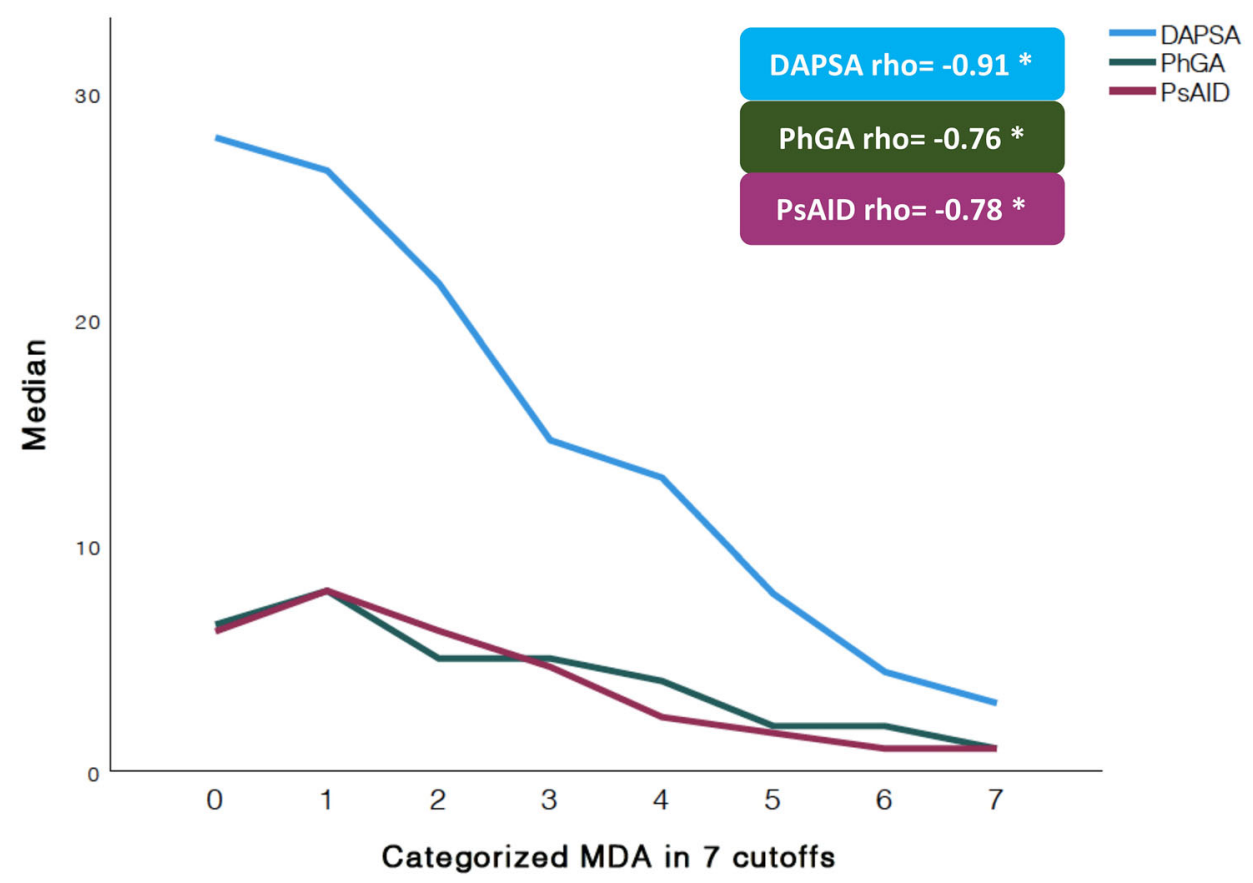

Fig. 2 Liner graph to show the correlation between the MDA divided in seven cutoffs with Disease Activity for Psoriatic Arthritis (DAPSA), Physician Global Assessment on VAS (PhGA), and Psoriatic Arthritis Impact of the Disease (PsAID). The relationship between MDA categorized in seven cutoffs (as continuous variable) and DAPSA,

MDA showing the importance to know what that remaining activity consists of.

Our study has strengths as well as limitations: the study was performed in a group of patients in a stable treatment and with a crosssectional design. At the same time, we did not perform any analysis on potential treatment implications in those patients (such as change therapy) due to the study design. However, our study tried to look at the differences in the MDA domains and, as far as we know, this is a novelty in this intriguing topic. Moreover, our patients seem to have a good control of the disease, with most patients having less than three swollen/tender joints and very mild skin involvement. This might explain why skin criteria and enthesis criteria were two of the most frequently fulfilled domains in every category of criteria fulfilled. Other studies partially agree with our results, in particular, Marin J et al. showed that skin was one of the major domains that stopped patients from achieving MDA. In our clinic,
PhGA, and PsAID is shown by Spearman's rho value. $D A P S A$ disease activity for psoriatic arthritis, $P h G A$ physician global assessment, $P S A I D$ psoriatic arthritis impact of the disease, $M D A$ minimal disease activity. *All the correlations had a $p<0.001$

patients are followed up regularly and treated to target, and this could explain the results obtained. However, as a possible further explanation is that at least $30 \%$ of patients of our group were under IL-12/23, Il-17 inhibitors [20].

\section{CONCLUSIONS}

In conclusion, this study detailed which domains are more frequently achieved on MDA divided into the seven cutoffs, underlying that "physician-driven" domains are more frequently achieved in all patients enrolled. Moreover, a strong correlation was found with other outcome measures throughout the seven domains.

\section{ACKNOWLEDGEMENTS}

We want to thank the participants in the study. 
Funding. No funding or sponsorship was received for this study or publication of this article. All authors had full access to all of the data in this study and take complete responsibility for the integrity of the data and accuracy of the data analysis.

Authorship. All named authors meet the International Committee of Medical Journal Editors (ICMJE) criteria for authorship for this article, take responsibility for the integrity of the work as a whole, and have given their approval for this version to be published.

Authorship Contributions. All authors have made substantial contributions to all of these sections: conception and design of the study, acquisition of data, analysis and interpretation of data, drafting the article, revising it critically for important intellectual content and final approval of the version to be submitted.

Disclosures. Ennio Lubrano, Silvia Scriffignano, and Fabio Massimo Perrotta have nothing to disclose.

Compliance with Ethics Guidelines. All procedures performed in studies involving human participants were in accordance with the ethical standards of the institutional and/or national research committee and with the 1964 Helsinki Declaration and its later amendments or comparable ethical standards. Informed consent was obtained from all individual participants included in the study. The study was approved by the Institutional Review Board of the University of Molise.

Data Availability. The datasets generated during and/or analyzed during the currentstudy are not publicly available but are available from the corresponding author on reasonablerequest.

Open Access. This article is licensed under a Creative Commons Attribution-NonCommercial 4.0 International License, which permits any non-commercial use, sharing, adaptation, distribution and reproduction in any medium or format, as long as you give appropriate credit to the original author(s) and the source, provide a link to the Creative Commons licence, and indicate if changes were made. The images or other third party material in this article are included in the article's Creative Commons licence, unless indicated otherwise in a credit line to the material. If material is not included in the article's Creative Commons licence and your intended use is not permitted by statutory regulation or exceeds the permitted use, you will need to obtain permission directly from the copyright holder. To view a copy of this licence, visit http://creativecommons.org/licenses/by$\mathrm{nc} / 4.0 /$.

\section{REFERENCES}

1. Ritchlin CT, Colbert RA, Gladman DD. Psoriatic arthritis. N Engl J Med. 2017;376:957-70.

2. Lubrano E, Scriffignano S, Perrotta FM. Psoriatic arthritis, psoriatic disease, or psoriatic syndrome? J Rheumatol. 2019;46:1428-30.

3. Tucker LJ, Coates LC, Helliwell PS. Assessing disease activity in psoriatic arthritis: a literature review. Rheumatol Ther. 2019;6:23-32.

4. Coates LC, Fransen J, Helliwel PS. Defining disease activity in psoriatic arthritis: a proposed objective target for treatment. Ann Rheum Dis. 2010;69: 48-53.

5. Coates LC, Helliwell PS. Defining low disease activity states in psoriatic arthritis using novel composite disease instruments. J Rheumatol. 2016;43:371-5.

6. Coates LC, Nash P, Kvien TK, et al. Comparison of remission and low disease activity states with DAPSA, MDA and VLDA in a clinical trial setting in psoriatic arthritis patients: 2-year results from the FUTURE 2 study. Semin Arthritis Rheum. 2020;4: 709-18.

7. Lubrano E, De Socio A, Perrotta FM. Comparison of composite indices tailored for psoriatic arthritis treated with csDMARD and bDMARD: a cross-sectional analysis of a longitudinal cohort. J Rheumatol. 2017;44:1159-64.

8. de Vlam K, Steinfeld S, Toukap AN, BEPAS Study Investigators, et al. The burden of psoriatic arthritis in the biological era: data from the Belgian Epidemiological Psoriatic Arthritis Study (BEPAS). 
Rheumatology (Oxford). 2021. https://doi.org/10. 1093/rheumatology/keab233.

9. Lubrano E, Scriffignano S, Azuaga AB, Ramirez J, Cañete JD, Perrotta FM. Assessment of the Patient Acceptable Symptom State (PASS) in psoriatic arthritis: association with disease activity and quality of life indices. RMD Open. 2020;6: e001170.

10. Gossec L, de Wit M, Kiltz U, EULAR PsAID Taskforce, et al. A patient-derived and patient-reported outcome measure for assessing psoriatic arthritis: elaboration and preliminary validation of the Psoriatic Arthritis Impact of Disease (PsAID) questionnaire, a 13-country EULAR initiative. Ann Rheum Dis. 2014;73:1012-9.

11. Schoels M, Aletaha D, Funovits J, Kavanaugh A, Baker D, Smolen JS. Application of the DAREA/ DAPSA score for assessment of disease activity in psoriatic arthritis. Ann Rheum Dis. 2010;69:1441-7.

12. Taylor W, Gladman D, Helliwell P, Marchesoni A, Mease P, Mielants H. Classification criteria for psoriatic arthritis: development of new criteria from a large international study. Arthritis Rheum. 2006;54: 2665-73.

13. Healy PJ, Helliwell PS. Measuring clinical enthesitis in psoriatic arthritis: assessment of existing measures and development of an instrument specific to psoriatic arthritis. Arthritis Rheum. 2008;59: 686-91.
14. Fredriksson T, Pettersson U. Severe psoriasis-oral therapy with a new retinoid. Dermatologica. 1978; 157:238-44.

15. Ranza R, Marchesoni A, Calori G, et al. The Italian version of the Functional Disability Index of the Health Assessment Questionnaire. A reliable instrument for multicenter studies on rheumatoid arthritis. Clin Exp Rheumatol. 1993;11:123-8.

16. Lubrano E, Perrotta FM, Parsons WJ, Marchesoni A. Patient's global assessment as an outcome measure for psoriatic arthritis in clinical practice: a surrogate for measuring low disease activity? J Rheumatol. 2015;42:2332-8.

17. Schoels MM, Aletaha D, Alasti F, Smolen JS. Disease activity in psoriatic arthritis (PsA): defining remission and treatment success using the DAPSA score. Ann Rheum Dis. 2016;75:811-8.

18. Gorlier C, Orbai AM, Puyraimond-Zemmour D, et al. Comparing patient-perceived and physicianperceived remission and low disease activity in psoriatic arthritis: an analysis of 410 patients from 14 countries. Ann Rheum Dis. 2019;78:201-8.

19. Lubrano E, Scriffignano S, Perrotta FM. Residual disease activity and associated factors in psoriatic arthritis. J Rheumatol. 2020;47:1490-5.

20. Marin J, Acosta Felquer ML, FerreyraGarrot L, Ruta S, Rosa J, Soriano ER. Patients with psoriatic arthritis fulfilling the minimal disease activity criteria do not have swollen and tender joints, but have active skin. J Rheumatol. 2016;43:907-10. 